\title{
La atención de la salud en Argentina: Al gran pueblo argentino... ¿Salud? - Tercera entrega: Formación médica en Argentina - Flojos de papeles
}

Health care in Argentina: To the great Argentine people ... Health? -Third issue: Medical training in Argentina

Carlos García

\section{Resumen}

En una serie de entregas relacionadas, el autor describe mediante la utilización de viñetas utilizadas a modo de ejem'plo, los diferentes factores que hacen de la administración de salud argentina una "tragedia".

En esta entrega se describe la deficiencia de la formación médica y la falta de exámenes periódicos de certificación y recertificación como factores determinantes de un nivel de formación deficiente en la Argentina.

\section{Abstract}

In a series of related articles, the author describes, through vignettes utilized as examples, the different factors that turns the Argentinian health administration into a "tragedy".

This issue is oriented toward description of the deficiency in medical training and the lack of certification tests as determinant factors of a very poor medical formation in Argentina.

García C. La atención de la salud en Argentina: Al gran pueblo argentino... ¿Salud? - Tercera entrega: Formación médica en Argentina - Flojos de papeles. Evid Act Pract Ambul 2016;19(4):101-104

La formación de un buen médico requiere un largo proceso de aprendizaje de grado en la escuela de medicina o facultad, un intenso proceso de aprendizaje de posgrado en la residencia médica y un proceso de educación médica continua que durará toda la vida y dependerá de la intensidad de la práctica, el intercambio de conocimientos con otros colegas y la realización de cursos y programas de actualización, en Argentina muy librados a la voluntad de los profesionales.

En algunos países, además, la educación continua requiere certificación y recertificación. Estas instancias son periódicas y poco menos que obligatorias y están brindadas por entidades que actualizan permanentemente las bases teóricas, elaboran, toman y califican los exámenes y entregan la certificación.

En Estados Unidos, por ejemplo, Cardiología es una de la 20 subespecialidades de Medicina Interna. Para ser cardiólogo se requiere completar la escuela de medicina, completar los tres años de la residencia de medicina interna y completar luego una formación (fellowship) de tres años en cardiología. Esto, le permite a un médico atender pacientes en el área de cardiología sin supervisión de otro especialista. A su vez, Medicina Interna y Cardiología deben dar sus propios exámenes periódicos de certificación: el Board de Medicina Interna y el Board de Cardiología'.

Esta certificación significa que los profesionales han demostrado, ante sus pares y ante el público que tienen el juicio clínico, las habilidades y actitudes para proveer servicios de excelencia. Las organizaciones que se encargan de estas certificaciones no son sociedades médicas sino organismos independientes de evaluación y certificación y su compromiso y responsabilidad es tanto para con los profesionales como para con la sociedad en general.

Dicho en otras palabras, ser un médico certificado significa que se han aprobado todas las instancias que garantizan una buena formación profesional.

La residencia médica, el proceso de especialización, es probablemente, el método de aprendizaje de posgrado más eficaz y reconocido en el mundo.

En Argentina, la base de grado (la facultad) es fundamentalmente teórica. Se egresa de las distintas facultades de medicina con experiencia y habilidades prácticas muy limitadas. En los seis o siete años de formación, los estudiantes de medicina se sientan frente a los libros y adquieren, en el mejor de los casos, un sinnúmero de conocimientos teóricos de miles de temas, muchos de los cuales jamás utilizarán en la práctica médica y quedarán sepultados en sus mentes como conocimiento inerte.
Primero se estudia la constitución del ser humano normal. La embriología nos enseña la formación de un ser humano desde la concepción, hasta el alumbramiento, la anatomía nos enseña la estructura del ser humano normal; la histología, las células que forman esas estructuras anatómicas; la fisiología estudia el funcionamiento normal del ser humano. Nos enseña cómo circula la sangre, cómo late el corazón, cómo los riñones se encargan de depurar tóxicos, regular la presión, el volumen sanguíneo y los electrolitos; los pulmones llevar oxígeno a la sangre, los glóbulos rojos transportarlo y depositarlo, los glóbulos blancos defendernos de agentes extraños; el sistema endócrino se encarga del metabolismo, las funciones sexuales, el metabolismo del calcio, etcétera.

En el segundo ciclo se empiezan a estudiar las enfermedades, los medicamentos y los tratamientos médicos y quirúrgicos; también cada especialidad aborda las patologías y los métodos de diagnóstico y tratamiento de diferentes órganos y sistemas. En este segundo ciclo, el ciclo clínico, existe contacto con pacientes pero es básicamente efímero y sin el apremio de la responsabilidad. Los alumnos, simplemente se limitan a mirar lo que los otros hacen, muy lejos de la responsabilidad, la obligación y el compromiso de la asistencia directa.

Los cuatro o cinco años de residencia son el dulce calvario, el aprender haciendo, con mucha responsabilidad, con agotamiento, con adrenalina, estómago vacío y boca seca.

En esos años todos esos músculos que estudiamos, sus células, su funcionamiento, sus enfermedades y sus tratamientos se nos juntan arrebatadamente en una dramática realidad. No pocas veces, la vida de nuestro sujeto de estudio, el ser humano, depende de nosotros ${ }^{2}$.

\section{La formación de grado}

En la Argentina, a fines de los años ochenta existían siete facultades nacionales de medicina y dos facultades privadas. Actualmente suman 30 . La calidad de la formación no tiene indicadores y una presunta mayor accesibilidad de clases sociales menos pudientes a la educación universitaria se pone claramente en dudas al ver que los egresados de esas escuelas siguen siendo en su mayoría hijos de las clases más pudientes que pueden estudiar sin trabajar ${ }^{3}$. La oferta de recursos educativos, fundamentalmente para las clases más acomodadas está garantizada, mas no la calidad de la misma. En 1992 había 60.128 alumnos y hubo 3.575 egresados. En el año 2009 había en medicina un total de 69.617 alumnos y egresaron 5.793 médicos. Dicho en otras palabras, aumentó exponencialmente el número de facultades (21 facultades más) y solo un $38 \%$ el número de los egresados. 
Hernán Escudero tiene 32 años, estudió medicina en la Universidad Nacional de La Plata de donde egresó con título de médico el 27 de marzo de 2014. Está casado con Silvia Solís Mendoza, recientemente recibida de enfermera en una escuela privada. Tienen tres hijos. Hasta ahora los ingresos de la familia dependían del trabajo de Silvia en un sanatorio privado de una localidad cercana a La Plata y de trabajos varios e informales que hacía Hernán para costearse los estudios y cubrir las necesidades de la familia.

A Hernán se le plantea un dilema: hacer una residencia médica, entre el trabajo diario y las agotadoras guardias, le demandará unas 80 horas semanales mientras que emplearse en un sistema privado de emergencias y consultas domiciliarias haciendo guardias de 12 horas a razón de diez por mes le permitirá duplicar los ingresos que obtendría durante los cuatro años de residencia y le permitirá aparte, asistir como concurrente informal a diferentes servicios médicos de clínicas y hospitales del área metropolitana. Los Escudero Solís, casi no tienen dudas. La residencia es un camino demasiado exigente y muy poco remunerativo. Hernán se emplea en Cruz Azul Medicina Domiciliaria.

María Laura Casalz tiene 25 años y acaba de recibir el título de médica cirujana de la Universidad Nacional de Córdoba. Santiago, su novio también acaba de recibirse de médico y planea hacer la residencia en cirugía general en un hospital de comunidad de Buenos Aires. María Laura quiere ejercer como médica pero también quiere tener niños y dedicarles gran parte de su tiempo.

Las residencias y las guardias son incompatibles con una "vida normal", dice María Laura. Su padre un conocido especialista de Córdoba le consigue una concurrencia en dermatología, en el mismo hospital en el que Santiago hará la residencia. Formándose en dermatología, si bien no cobrará sueldo al principio, no tendrá que hacer guardias y podrá trabajar ocho horas diarias. Tanto los padres de María Laura como los de Santiago, contribuirán económicamente en los primeros años de formación de sus hijos.

En la Argentina solo un 50 por ciento de los médicos hace la residencia ${ }^{4}$. Muchas residencias son agotadoras al límite de lo inhumano.

La creciente demanda y mejor remuneración de la atención episódica, sea en guardias fijas, en guardias domiciliarias o en consultorios de demanda espontánea a los que concurren diariamente miles y miles de individuos de clase media y alta por cuadros que van desde resfríos comunes, confecciones de recetas, pedidos de exámenes de laboratorio vencidos o síntomas triviales e inespecíficos de pacientes que no tienen un médico de cabecera o que aun teniéndolo no disponen de la suficiente accesibilidad, va traccionando en forma continua y permanente a los jóvenes médicos hacia una formación informal, mejor remunerada y mucho menos exigente. También, mucho más precaria y de peor calidad, la medicina episódica, el contacto único, un escenario donde los pacientes se quejan de los médicos y los servicios y los médicos de la petulancia y frivolidad de estos pacientes golondrinas.

Los médicos en la Argentina de gran parte del siglo XX hicieron su formación en los hospitales públicos. De ellos salieron camadas y camadas de profesionales con una buena formación médica, o al menos, al estilo de sus maestros, sin mayores parámetros objetivos como para discernir qué es una "buena" formación médica.

Cirujanos, clínicos, neurólogos, cirujanos cardiovasculares, gastroenterólogos, ginecólogos, pediatras, cardiólogos y muchos otros especialistas más hicieron escuela y fijaron rumbos en la medicina Argentina que atrajo a sus claustros a jóvenes de toda Latinoamérica, fundamentalmente de Bolivia, Perú y Paraguay.

Pero esa excelencia académica obtenida en el seno de la salud pública generó un engendro difícil de resolver: la medicina privada, la medicina de mercado, una atención sanitaria que provee recursos en exceso a quienes tienen medios y acceso, y deja desprotegidos a grandes sectores poblacionales de menos recursos.

Así, los médicos se formaban, hacían "su mano" y su prestigio, por la mañana en el hospital público donde los casos simples y complejos desbordaban. Pacientes que viven los cuidados médicos recibidos más como un favor que como un derecho y obligación del Estado y la sociedad, que deberían asegurar que todos los individuos tengan oportunidad de acceso al mismo nivel y calidad de cuidados.

Progresivamente empezó el "descreme", la selección de casos más atractivos y rentables. Generaron a pocas cuadras, cuando no enfrente de los hospitales escuela, los centros privados de atención, diagnóstico y tratamiento donde con más tiempo, bonitos cuadros renacentistas en las paredes plagadas de cuadritos de congresos internacionales y enfermeras de guardapolvos inmaculados, podían atender a la gente pudiente "como era debido".

El hospital público financiado con recursos que salen de los bolsillos de toda la población forma y da prestigio a quienes cruzan la calle a sus consultorios privados donde atenderán a los que pagan.

\section{La asistencia domiciliaria - La imaginación llevada a la práctica}

A Flavia Casabé le parece estar transitando las diez plagas de Egipto. La empleada doméstica (muchas veces llamada "la muchacha" o "la chica que me ayuda") no vino a su trabajo porque llueve torrencialmente y un paro de choferes de transporte público reclamando por mayor seguridad ante la muerte de un chofer, convirtieron la ciudad en un infierno. Primero fue Sol, su nena de cinco años, la que trajo el resfrío del pre escolar, luego Pedro (3), Miguel (1) y ahora ella. "Esta casa es un hospital dice Flavia" mientras lee el 0800 de un imán adherido a la heladera por el que pedirá médico a domicilio.

A las cuatro horas (una vergüenza para Flavia) llega Hernán Escudero que ve a Flavia y de paso a los niños. Flavia se queja de sus mocos verdes y dolor en la cara, cabal conocedora de que si uno no dice que el moco se puso verde o que le empezó a doler la frente y la cara, o que se vio en la garganta unas plaquitas blancas, los médicos no le darán antibióticos. Al rato, la mamá de Flavia que dejó su taller de pintura para ayudarla, baja a la farmacia a comprar descongestivos nasales, expectorantes, antitusivos y un antibiótico que no tiene pertinencia pero está de moda en el tratamiento de las virosis respiratorias, la levofloxacina.

Hernán se va con la satisfacción de habérsela sacado de encima bastante rápido y el displacer de que al despedirse, Flavia no dejó de informarle que iba a hacer una queja a Cruz Azul por "la inadmisible demora en la atención".

La imaginación y el emprendimiento humano no tienen límites. El mercado de la salud es opulento y da para mucho. Un médico iluminado para los negocios crea a principios de los años 80 
un nuevo engendro: la asistencia médica domiciliaria. Así, por una módica cuota mensual, la clase pudiente podía tener a su merced, las 24 horas del día, una ambulancia, con un chofer, un médico y a veces un enfermero para atender cuadros que en el noventa por ciento no iban más allá de un resfrío o un dolor de espalda u oídos. Lo que se entendía por brindar calidad era llegar rápido a un piso de Barrio Norte de la Capital Federal, aunque su causa no fuera más que unas líneas de fiebre.

Estos centros privados satélites de los hospitales públicos y este mercado del resfrío se convirtieron primero, en un mercado complementario atractivo para los jóvenes que se formaban por pocos pesos y mucho esfuerzo en el hospital público y así, con poco esfuerzo y muchos más pesos, completaban sus ingresos. Aprendieron también a odiar a sus frívolos pacientes circunstanciales y también a sus empleadores. Aquéllos por demandarles altaneramente que acudan rápido a su domicilio a solucionarles problemas nimios; éstos por fijarle precio de mercado a su hora de trabajo.

La mejor forma de sacarse de encima un resfrío común que llama médico a domicilio, en la Argentina pudiente, es no minimizarlo, hablarle de un cuadro serio y darle descongestivos, analgésicos antipiréticos y antibióticos. La percepción de calidad por parte de los consumidores de medicina a medida pasa por la rapidez de la asistencia, los estudios ordenados y los medicamentos prescritos ${ }^{5}$

Una nueva vuelta de tuerca de esta "medicina al paso" es que ya no se envían ambulancias con médico, enfermero y chofer por cualquier cosa. Los financiadores compraron pequeños vehículos utilitarios en los que un chofer lleva el médico al domicilio; otros médicos trabajan con su propio auto.

La mayor parte de los resfríos de mis pacientes, de quienes soy médico de cabecera desde hace muchos años, puedo manejarlos por teléfono, sin estudios y sin antibióticos, antivirales ni antihistamínicos. Está hartamente demostrado que los antibióticos, los antivirales y los antihistamínicos no tienen ningún rol en los resfríos y que su uso indiscriminado lo único que logra es una creciente resistencia de los microbios. Mis pacientes tienen acceso a mí, me conocen y confían en mí, saben que ir a una sobrecargada central de emergencias o llamar un médico a domicilio por un resfrío, por más fuerte que éste sea, son motivos de excomunión.

\section{La formación de posgrado en los centros públicos de forma- ción - Una vaca lechera con poco pasto para alimentarse.}

\section{Certificación y revalidación de la licencia profesional}

El proceso de diagnóstico ha evolucionado dramáticamente a lo largo de las décadas. La larga tradición de "ver uno, hacer uno, enseñar uno", seguida por estudiantes y maestros altamente motivados demostró ser exitosa. En las últimas cuatro décadas los avances tecnológicos crecieron de una manera tan exponencial que hicieron que gran parte de la habilidad deba incluir la expeditiva administración de los recursos diagnósticos utilizando el menor número posible de procedimientos caros y riesgosos a la vez.

El diagnóstico es un proceso inferencial llevado a cabo en situaciones de incertidumbre y la simple utilización indiscriminada de múltiples exámenes es peligrosa e injustificada. Contrariamente a la creencia popular que entiende que "más es mejor" en la realización inapropiada e irracional de exámenes médicos "más, suele ser peor".
Mauricio Cohen tiene 57 años. Se recibió de médico con diploma de honor en la Universidad de Buenos Aires el 23 de diciembre de 1983 cuando la Argentina acababa de salir de una larga y aterradora noche. Se recibió con Medicina III, el propio Profesor Titular, el doctor Fernando Díaz Troiani, lo felicitó por su brillante examen y lo invitó a que venga un día a charlar con él en la Cátedra. Ese día lo tentó a que haga la residencia de clínica médica. Mauricio no dudó, ya en febrero empezó a ir al gigantesco y prestigioso aunque bastante derruido hospital escuela. Subir por escalera los ocho pisos los no pocos días en que no funcionaba el ascensor lo hacían sentirse el elegido que con su antorcha subía a encender la llama olímpica. Había rendido 36 exámenes en la facultad en forma brillante, se recibió con un promedio de 8,76 , entró cómodo en la mayoría de los centros en que se postuló para ser residente y hoy, empezaba a ser uno de los elegidos de Díaz Troiani al que acompañaría, como su fiel mano derecha, hasta su muerte. Seguiría en la Tercera Cátedra, vería los pacientes de su consultorio privado y también terminaría comprando algunas acciones del centro de diagnóstico y de Cruz Azul, la empresa de emergencias domiciliarias.

Su último examen "serio" fue el de las residencias. Nunca más se le exigió ningún tipo de certificación.

Nadie duda de la capacidad de Mauricio. Las dos o tres horas que pasa en el Hospital Escuela lo exponen al fuego cruzado de la ciencia creciente. Enfrente, está su consultorio privado. Pronto, y mientras el cada vez más exhausto Hospital Escuela resista, tendrá una nueva réplica de su modelo.

En la Argentina, el único requisito importante para ejercer la medicina es haber dado los exámenes curriculares de la carrera, en general, unas 36 materias. La residencia no es obligatoria y un creciente número de egresados sortea esta pesada opción por la menos exigente y más tentadora de la asistencia informal.

Un hecho que siempre me resultó curioso y anecdótico es el título con el que egresan los médicos de la Universidad Nacional de Córdoba: Médico Cirujano, cuando a duras penas un egresado de cualquier facultad de medicina del país podría hacer una simple sutura de una herida cortante lineal y no complicada.

Probablemente el principal proveedor de médicos especializados, con residencia de relativa calidad en la Argentina, es el hospital público. Con recursos limitados los hospitales públicos, aseguran a los médicos en formación una importante intensidad de servicios y entrenamiento suficiente provisto por parte de profesionales ya formados para poder ejercer una profesión y especialización de calidad.

Sin embargo, la residencia no es obligatoria, tampoco existen exámenes serios y exigentes de certificación y recertificación. El número de centros que organizan residencias médicas en Argentina es creciente y suficiente para que todos los egresados de sus escuelas de medicina puedan tener algún tipo de formación de posgrado. Sin embargo, la calidad de esas residencias es muy irregular y muchos centros privados crean residencias como una forma de asegurarse mano de obra intensiva a bajo precio.

Cualquier médico que realice algunos trámites burocráticos o haya realizado alguna pasantía en algún servicio o especialidad puede registrarse en cualquier región del país y ejercer cualquier especialidad que sea de su interés o necesidad de la región.

La certificación y revalidación del título no es necesaria en Argentina. Cualquier médico puede ejercer libremente la profesión sin necesidad de dar un solo examen más fuera de los exámenes de las materias curriculares de la carrera de medicina.

El instrumento más objetivo que tiene la sociedad argentina 
para evaluar la capacidad de un profesional suelen ser, los consejos de la gente o de algunos colegas, el lugar donde ejerce y muchas veces, la cantidad de cuadritos de asistencia a congresos mundiales pagada por la industria farmacéutica o tecnológica.

\section{Reino Unido - Algunas comparaciones}

En Inglaterra la provisión, estructuración, asignación y planificación de recursos están fuertemente reguladas ${ }^{6}$.

Inglaterra tiene en 2016 alrededor de 55 millones de habitantes.

Existen en Inglaterra 22 escuelas de medicina con amplia distribución geográfica capaces de asegurar la educación médica apropiada para las necesidades de su población.

La distribución de los médicos está regulada de acuerdo a las necesidades regionales. Los médicos están sujetos a revalidaciones y recertificaciones de especialidades para renovar su licencia. Este proceso de renovación se realiza cada cinco años.

La atención primaria en Inglaterra es la fuente de un amplio rango de servicios mayormente provistos por médicos generalistas. Los médicos generalistas son el primer punto de contacto para un individuo y manejan la mayoría de los problemas médicos que un paciente tiene a lo largo de su vida con la habilidad y capacidad para referir a los pacientes a niveles más especializados cuando es necesario. Los médicos de atención primaria trabajan en el ámbito ambulatorio. Los especialistas tienen su práctica en los hospitales.

La distribución geográfica de los profesionales de la salud en Inglaterra está regulada de manera que sea capaz de asegurar una distribución equitativa de los recursos.

Dicho en otras palabras, ser médico en Inglaterra requiere una estricta formación y certificación, luego formación continua, recertificación y radicación en los lugares en los que el Sistema Nacional de Salud lo requiera.

\section{Conclusiones}

La provisión de servicios de salud en la Argentina está sumamente desregulada, atomizada y librada a la voluntad de los prestadores y las fuerzas del mercado.

Uno de los problemas importantes es la deficiente formación de los profesionales médicos que pueden ejercer libremente sin necesidad de una formación específica, certificación ni recertificación periódicas.

La especialización de los profesionales no es rigurosa, tampoco requiere certificación ni recertificación y sigue las leyes de mercado.

Los hospitales públicos siguen siendo la fuente principal de formación médica, sin embargo la mayor parte de los ingresos en el sector salud se los lleva la medicina privada.

Recibido el 18/12/2016 y aceptado el 20/12/2016.

\section{Referencias}

1. American Board of Internal Medicine. American Board of Internal Medicine [Internet]. Available from: https://www.abim.org/about/default.aspx 2. Duré I. RESIDENCIAS MÉDICAS EN ARGENTINA 2015. Rev Argentina Med [Internet]. 2015;3(5):2-4. Available from:

http://www.msal.gob.ar/observatorio/images/stories/documentos formacion/acerca_de/Articulo Residencias_Medicas en la_Argentina.pdf

3. AA G. Inclusión social para la UBA. La Nación [Internet]. Ciudad Autónoma de Buenos Aires; 2013 Feb 8; Available from: http://www.lanacion.com.ar/1552785-inclusionsocial-para-la-uba

4. Valeria Román. Se reciben 6.000 médicos y la mitad no hace la residencia. Clarín [Internet]. Ciudad Autónoma de Buenos Aires; 2012 Jun $25 ;$ Available from: http://www.clarin.com/sociedad/reciben-medicos-mitad-hace-residencia_0_725327510.html

5. Porter ME. What Is Value in Health Care? N Engl J Med [Internet]. 2010 Dec 23 [cited 2016 Dec 21];363(26):2477-81. Available from: http://www.ncbi.nlm.nih.gov/pubmed/21142528

6. Cylus J, Richardson E, Findley L, Longley M, O’Neill C SD. United Kingdom Health system review. Health Syst Transit [Internet]. 2015;17(5):1-125. Available from: http://www.euro.who.int/_data/assets/pdf_file/0006/302001/UK-HiT.pdf?ua=1
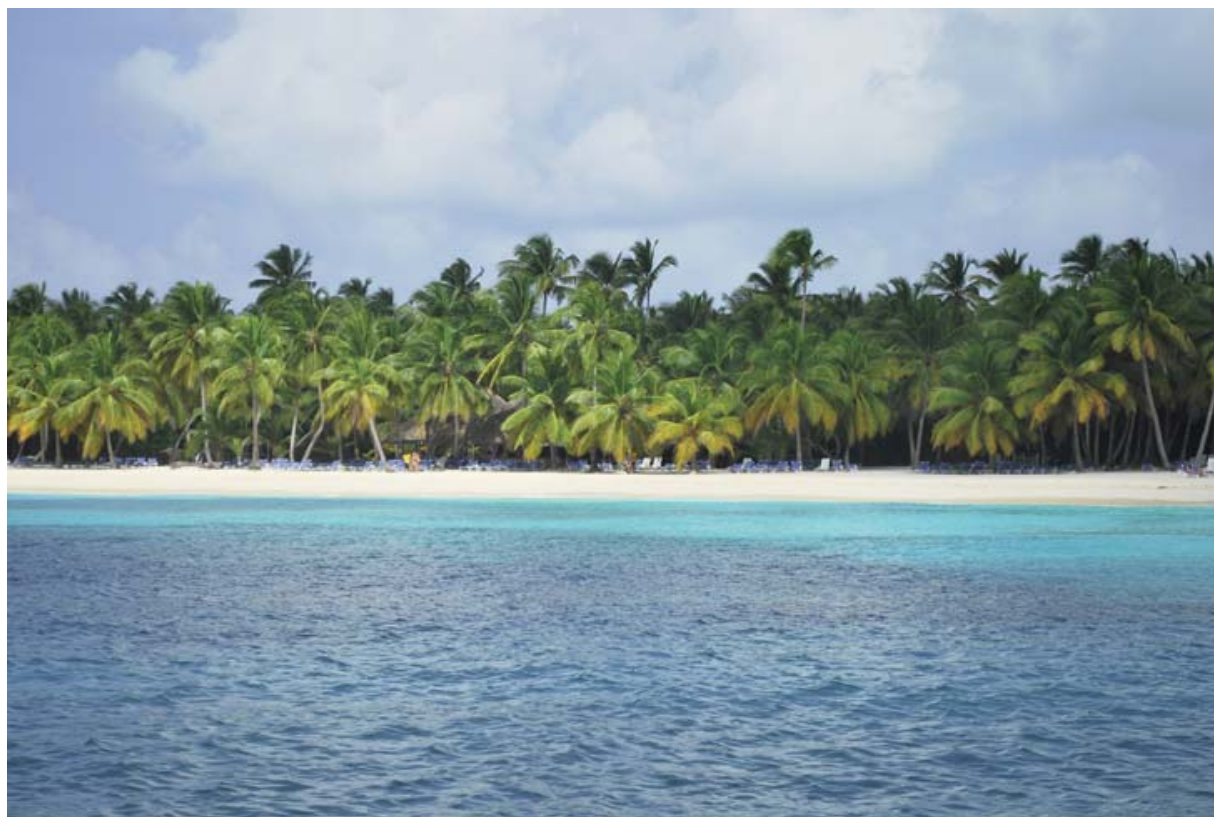

Cortesía: Silvia Spina 